\title{
Lifestyle Impact on Cognitive Decline and Neurodegeneration Overview
}

\author{
Alexander Victor Anand David ${ }^{1 *}$, Subramani Parasuraman²
}

\section{Alexander Victor Anand David ${ }^{1 *}$, Subramani Parasuraman $^{2}$}

'Unit of Anatomy, Faculty of Medicine, AIMST University, Semeling, Bedong, Kedah, MALAYSIA.

'Unit of Pharmacology, Faculty of Pharmacy, AIMST University, Semeling, Bedong, Kedah, MALAYSIA.

\section{Correspondence}

Dr. Alexander Victor Anand David

Unit of Anatomy, Faculty of Medicine, AIMST University, Semeling, Bedong08100, Kedah, MALAYSIA.

Phone no: +60 0124771109

E-mail: drvdavid7@gmail.com

\section{History}

- Submission Date: 21-08-2019;

- Review completed: 06-09-2019;

- Accepted Date: 05-10-2019.

DOI : 10.5530/fra.2019.2.10

\section{Article Available online}

http://www.antiox.org

\section{Copyright}

(c) 2019 Phcog.Net. This is an openaccess article distributed under the terms of the Creative Commons Attribution 4.0 International license.

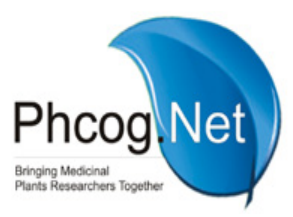

\begin{abstract}
Cognitive decline is a part of a natural phenomenon that eventually happens to almost everyone as they age. It results from a combination of lifestyle habits, genetic factors, trauma or just gradual wearing-out of one's nervous system. The effects of lifestyle and behavior which include patterns of dietary intake, physical activities as well as practicing unhealthy habits such as smoking and alcohol consumption, have been globally emphasized and embraced time and again as a crucial component to avoid serious health complications. It is also been included as an evidence-based guideline for children, adolescents and adults from diverse populations in all countries. Healthy lifestyle behaviors and therapeutic lifestyle changes were emphasized and reaffirmed as central to cardiovascular health promotion and risk reduction, respectively. Certain healthy behaviors known to combat cancer, cardiovascular disease, diabetes and may also reduce the risk of cognitive decline. Simple lifestyle changes might delay the start of cognitive decline and dementia or slow its progression. Activities that boost your body and brain are such as staying mentally active, engaging in regular physical activity and eating a heart-healthy diet. There are also evidences where people may benefit from staying socially engaged with friends, family and the community. People can reduce the risk of cognitive decline by making key lifestyle changes. There are measures which can be taken to modify their lifestyle while they still can before it's too late to stop if not to minimize the onset of neuron degeneration.

Key words: Alzheimer's disease, Lifestyle, Neuron degeneration, Cognitive decline, Dementia, Neurological deficit.
\end{abstract}

\section{INTRODUCTION}

The term lifestyle refers to day to day behaviours, habits and functions of an individual involving their activities, job and diet. It is a way used by people from various countries or referred to the characteristics of inhabitants from a specific geographical region, economic, political, cultural and religious practices. ${ }^{1}$ According to WHO in recent years, lifestyle behavioral pattern and habits are said to be one of the contributing factors for the onset of various metabolic diseases which can lead to serious life-threatening health conditions. It is said that more than $50 \%$ of related factors affecting the individual health and quality of life are correlated to lifestyle. ${ }^{2}$ Over the past decade, with the emerging of new technologies such as the internet and virtual communication networks have led our world to a major challenge that threatens the physical and mental health well-being of millions of people. In order to keep up with the growing tech world, the interest in younger generation is strongly shifted to learning new things out of curiosity and most people do not realize that this pathway follows an unhealthy sedentary lifestyle. The link between lifestyle and general health should be highly considered as many studies revealed that amid acquiring knowledge on new gadgets and technologies, most

people get their focus deviated, eventually being unaware of the health consequences. It is evident that recent technologies which include electronic games, home computers, handheld devices, smartphones, iPods etc. play a vital role in our daily lives these days. Gadgets are popular in children as well as elders. ${ }^{3}$ It is indeed a sad reality to embrace and to say most people encounter illnesses, disabilities and even death at an early age nowadays as compared to the previous generations. Health problems like metabolic diseases, cancer, joint and skeletal problems, cardiovascular diseases, diabetes, obesity and so on, can be caused by unhealthy lifestyle habits, addiction and physical inactivity. ${ }^{4}$ According to a research done by Brannon and Feist, fatalities in the $20^{\text {th }}$ and $21^{\text {st }}$ century results from diseases strongly associated with individual lifestyle/behavior. ${ }^{5}$ In addition to habits, there have been many studies done on sedentary lifestyle being strongly linked with the onset of various metabolic diseases. The term cognitive decline refers to deterioration in memory or cognition which may take place as a normal process. Various research studies have revealed that lifestyle and habits can be directly related to the onset of cognitive decline leading to certain neurodegenerative conditions. Although 
there may not be any specific lifestyle or habits which trigger cognitive neuron cell death, it may be more appropriate to mention that this works accumulatively over a long period of time in certain individuals. Most often we are aware of the known fact that diseases are said to be associated with the type of diet and hereditary factors. Lifestyle defines a person and the kind of consequences that comes is a package which they have to deal with that may or may not be reversed according to the damage sustained.

\section{Importance of Lifestyle and Behaviour}

The common factors contributing to sedentary lifestyle are socio-economic status, age and gender, advancement in technologies and long working hours. Quality of life is the result of diverse factors and behavior/lifestyle is one of the most powerful determinants of good health. ${ }^{6}$ Most people do not realize this correlation which eventually may or may not progresses to cognitive decline and other types of neurodegenerative conditions. In recent decades, researches have taken interest in lifestyle and habits as being an important factor of health and the onset of diseases. According to WHO, $60 \%$ of related factors to individual health and quality of life are correlated to lifestyle. ${ }^{2}$ In recent days, we see extreme changes taking place in the lives of people regardless of age, gender and personal backgrounds. Malnutrition, unhealthy diet, smoking, alcohol consumption, drug abuse, stress and so on, are the presentations of unhealthy lifestyle that has been known to be the dominant forms of lifestyle.

The concept of sedentary behavior is a term used to characterize those behaviors that are associated with low energy expenditure. The definition of "Sedentary" in Latin which is "Sedere" means "to sit". This includes prolonged sitting at work, home, business centers, long screen time, car driving and leisure time. ${ }^{7}$ Despite the well-known and discussed benefits of physical activity, many adults and children continue to lead a sedentary lifestyle. It has been revealed that most urban dwellers choose to adapt sedentary lifestyles which have obvious negative health implications particularly cardiovascular diseases. ${ }^{8}$ The importance of healthy lifestyle behaviors has been universally emphasized and embraced as a central component of evidence-based guidelines for children, adolescents and adults from diverse populations. Health behaviors, including patterns of dietary intake, physical activity and inactivity, substance abuse like drug addiction, smoking and alcohol consumption are often one of the major root causes of serious illnesses. These unhealthy behaviors have led to various chronic diseases such as heart diseases, deep vein thrombosis, Type-2 diabetes, hypertension, osteoporosis, cancer, muscle and skin disorders also neurodegenerative diseases. It is a known fact that these diseases are among others with resultant increase in morbidity and mortality. ${ }^{9}$

Previous studies and evidences showed that lack of physical activity ameliorates psychological stress, vascular and metabolic risk factors (such as high blood pressure and diabetes)..$^{10,11}$ Frequent engaging in physical activity can also bring about amyloid clearance and increase the cognitive reserve, which is defined as the brain's ability to cope with damage and still function adequately. ${ }^{12}$ Increased brain volume and elevated levels of brain-derived neurotrophic factors have also been closely associated with physical activity.13,14

\section{Variables of Lifestyle}

The concept of quality of life indicates health and functional status, perceived health status, subjective health, health perception, symptoms, satisfaction, cognition, functional disability, disorder and well-being. ${ }^{15}$ Quality of living varies from each individual based on the circumstances and area of interest, ranging from those associated with the basic necessities of life, to the realization of feelings and satisfaction, even including physical, psychological and social dimensions. ${ }^{16-18}$ It has already been proven that having a systematic quality life with strict diet monitoring can minimize certain types of diseases. Some of the common variables of lifestyle that influence health can be categorized as the following items:

1. Exercise: This has been globally accepted and established fact through various studies that exercise plays a crucial role if not treating but minimizing general health problems. ${ }^{19}$ Continuous and regular exercise along with a healthy diet increase the health benefits especially cardiovascular circulatory. Some studies stress on the relation of active lifestyle with happiness. ${ }^{20,21}$ Physical activities produces beneficial effects through mechanisms that involve the physiology of the cardio circulatory system, glucose and lipid metabolism in general and the coagulation process. The present orientation is that moderate daily physical activity such as walking or biking for 30-45 min a day has the exact outcome as performing more vigorous physical activities.

2. Sleep: The quality of sleep holds a significant key to a healthy life. Sleep disorders have been linked to several social, psychological unhealthy consequences. The nature of a person's lifestyle may affect the quality of sleep and this has a clear influence on mental and physical health, which may lead to neuron degeneration. Problematic or disturbed sleep has adverse effects on all individuals, regardless of age and gender; however, older people typically show an increase in disturbed sleep or reduced sleep patterns that can create a negative impact on their quality of life, mood and alertness. ${ }^{22,23}$ Therefore, regular exercise and good quality of sleep are excellent combination to maintain a good health not merely physically but mentally as well.

3. Body Mass Index and Diet: Poor diet is also an important factor in daily lifestyle and has a direct and positive relation to health. The consequences of unhealthy diet are one of the common issues in urban societies. Body Mass Index (BMI) is the key measurement to monitor unhealthy lifestyle. Vegetables may also have a neuroregulatory role by inducing satiety and ultimately reducing the risk of obesity, which is another major risk factor for accelerated cognitive decline and dementia. ${ }^{24}$ Urban lifestyle due to limited time and feasibility leaves most people with no option but to settle in for a quick bite turning to good old fast foods which is a growing trend among younger generations especially with some of the outlets offering free Wi-Fi for the customers. This is not only common amongst students and youngsters but even amidst the business-oriented people in which they have their regular meetings in these outlets. This goes on to develop imbalanced nutrition problems and increasing cardiovascular conditions. ${ }^{25}$ It is now evident that the intestinal microbes influence host function well beyond the gut and it has been implicated in a variety of diseases including obesity, diabetes, non-alcoholic fatty liver disease, autism, multiple sclerosis and cardiovascular disease. ${ }^{26}$ In recent research work, it was found that cognitive decline in hepatic encephalopathy is associated with the presence of specific bacteria, although there was no causality reported. ${ }^{27}$ Diet is said to be an important factor which will impact on the composition of the systemic milieu and can similarly modulate stem cell ageing, adult hippocampal neurogenesis and cognition. ${ }^{28}$ Therefore, modulation of adult hippocampal neurogenesis by diet emerges as a possible mechanism by which nutrition can impact on cognitive ageing either via the systemic milieu or via the gut-microbiota, as discussed earlier.

4. Substance and medication abuse: The word abuse is related to an addiction of any substance, which brings adverse events/reactions to the human body which is considered as an unhealthy lifestyle. Smoking, drug addiction and alcohol consumption are the main substance that may result in various problems like cardiovascular disease, 
asthma, liver diseases, cancer, brain injury, etc. One of the longitudinal studies has indicated that $30 \%$ of people between the ages of 18-65 years old, who smoke cigarette permanently, ultimately lead to chronic illnesses. ${ }^{29}$ In addition to that, using normal medication as self-treatment, sharing medication, using medications without prescription, prescribing too many unnecessary drugs which may be harmful on prolong use goes on to disrupt the normal functioning of several organs. Drug abuse, as a social phenomenon, is one of the chronic problems of the present era that almost all the countries are dealing with globally. It has been known to have social, psychological, physical and economic effects. ${ }^{30}$ The amount of drug abusers varies in different countries, races commonly in young adults and currently it is seen even in much younger generation. The range of suicidal tendencies has been very high due to drug abuse with an increased amount of early deaths. ${ }^{31,32}$ Chronic drug addictions in any form and excessive alcohol consumption is a known fact for the onset of neuronal cell death which eventually leads to cognitive decline and neurodegeneration. ${ }^{34}$ It was also reported that drug abuse has a significant relationship with the level of education. Lower degree of education obviously has inadequate knowledge about drugs and their effects. ${ }^{33,34}$

5. Modern technologies: Advanced technology and facilities in the current era and the misuse of technologies often result in unpleasant consequences. For example, using of computer and other devices all through the day up to midnight to wee hours of morning may affect the pattern of sleep and eye conditions. It has been reported that mobile phone addiction is related to symptoms of depression and neurological hazards due to constant exposure to radiation. ${ }^{35}$ Though it has become a necessity to keep up with the tech world and devices, unfortunately this unhealthy lifestyle can result in detrimental effects

6. Recreation: Outdoor activities, socializing, travelling or leisure time with hobbies is a significant key factor which must be included as part of our lifestyle. This is seen usually in the urban community which reflects on how important recreation in one's life even before the onset of certain common and terminal diseases. It is a normal phenomenon whereby neglecting leisure or any form of recreation can bring negative consequences on physical and mental health. On the other hand, with disorganized planning, unhealthy leisure and lifestyle, people sometimes do endanger their health as well. ${ }^{36}$

7. Ethnicity and Socio Economic Status: Socio-Economic Status (SES) such as parental income and education, are inversely associated with sedentary behavior. The sedentary behavior tends to be higher in low SES groups as their knowledge is very limited on health and factors affecting the human body. ${ }^{37}$

8. Sedentary lifestyles: Reduced physical activity which characterizes sedentary lifestyle results in accumulation of excess calories and fatty acids. An individual, who leads a sedentary life, absorbs and stores a lot of calories because of reduced energy expenditure. ${ }^{38,39}$ These unwanted calories leads to obesity. It is an unhealthy condition associated with excess body fat. ${ }^{40}$ Obesity has been identified as one of the emerging major problems of younger age groups and adults especially those in urban areas and developing countries due to availability of amenities and over-eating tendencies. Health problem due to obesity carries a very high morbidity and mortality rates. Some of the diseases arising from sedentary lifestyle are type-2 diabetes, hypertension, hypercholesterolemia, cancer, cardiovascular diseases, muscle and skin changes and vitamin deficiency. It has been revealed that 9 out 10 type- 2 diabetes could be prevented if some of the risk factors were eliminated, these include-overweight, unhealthy diet, smoking and physical inactivity. ${ }^{41}$ Salmon $\mathrm{J}$ et al. discovered that increased sitting time exposed most people to type- 2 diabetes. WHO correlated that prolonged sitting time or inactive stage due to sedentary lifestyle increases great risk of developing colon cancer in men whereas endometrial and breast cancer in women. ${ }^{42}$ Muscle fibers also undergo changes with prolonged sitting. One begins to lose the muscle fibers that are responsible for active movements. Hypercholesterolemia causes $18 \%$ of global cerebro vascular disease and $56 \%$ of ischemic heart disease.$^{43}$ If muscles are not used constantly with regular exercise, the fibers become gradually replaced with fat and muscle wasting eventually occur. ${ }^{44}$

\section{Neurodegeneration and Cognitive Decline}

It is predicted that by 2050 , there will be an increase in the ageing population presenting significant economic, social challenges, burden for healthcare systems and on society will rise. According to the studies, by 2050, the likelihood of developing neurodegenerative diseases, such as Alzheimer's or Vascular Dementia (VD) will increase by $45 \%{ }^{45}$ Cognitive function tends to improve over the first 18 years of life, peak during the next few years and then deteriorate steadily thereafter. ${ }^{46,47}$

Neurodegenerative diseases result from gradual or progressive loss of structure and function which in the end leads to cell death of neurons (cells responsible for the nervous system functions). This degeneration can affect movements of the body and brain function, with or without causing dementia (progressive or chronic decline of cognitive function that affects memory, thinking, behavior, calculation, learning and emotion capacity and should never be associated with a normal aging process). ${ }^{16,48}$ The major problem is that these disorders are normally detected behindhand and restricting the efficacy of the treatment options. Neurodegenerative diseases have major impact at professional, social and family level that can lead to a complete inability to carry out any type of daily activity. Some of the common associated signs are motor, breathing difficulties, cognitive problems or gradual memory loss (possibly affecting the memory of all that has been learned over a lifetime).

The brain is affected in several ways in the process of aging, from the cellular to functional level. Changes associated with age manifest themselves as decline and deterioration in several abilities, including sensory, motor and higher cognitive functions. ${ }^{49,50}$ Most evidently, there has been certain specific diseases strongly related to age causing lesions in the brain that exacerbate the physiological changes which occur during normal aging. Cognitive decline is a classic example of this interaction between aging-related changes and age-related diseases in older people. The decline includes a large spectrum of clinical manifestations, which ranges from intact cognition through mild cognitive impairment (MCI) and finally, dementia. The term dementia is characterized by progressive deterioration in multiple cognitive domains that is severe enough to interfere with daily functioning. ${ }^{51}$

Although the most consistent risk factor for developing a neurodegenerative disorder, especially $\mathrm{AD}$ or $\mathrm{PD}$, some cognitive decline is expected with age, the reason is not yet known on how this may directly relate to dementia. Neurodegenerative diseases are multifactorial debilitating disorders of the nervous system that affect approximately 30 million individuals worldwide (Table 1). Neurodegeneration is not an acute or sudden phenomenon but a gradual process which involves many factors and lifestyle habits is most certainly is a part of contributing factor. $^{52}$ Alzheimer's, Parkinson's, Huntington's and Amyotrophic Lateral Sclerosis make up a group of neurodegenerative diseases which are characterized by specific etiologies with different morphological and pathophysiological features. It is the consequence of misfolding and dysfunctional trafficking of proteins. ${ }^{53}$ Reactive oxygen species (ROS) which are naturally generated chemical molecules within the biological system have been implicated in the pathogenesis of neurodegenerative diseases. It plays an important role in mediating cellular activities such as inflammation, cell survival and stressor responses as well as many diseases 


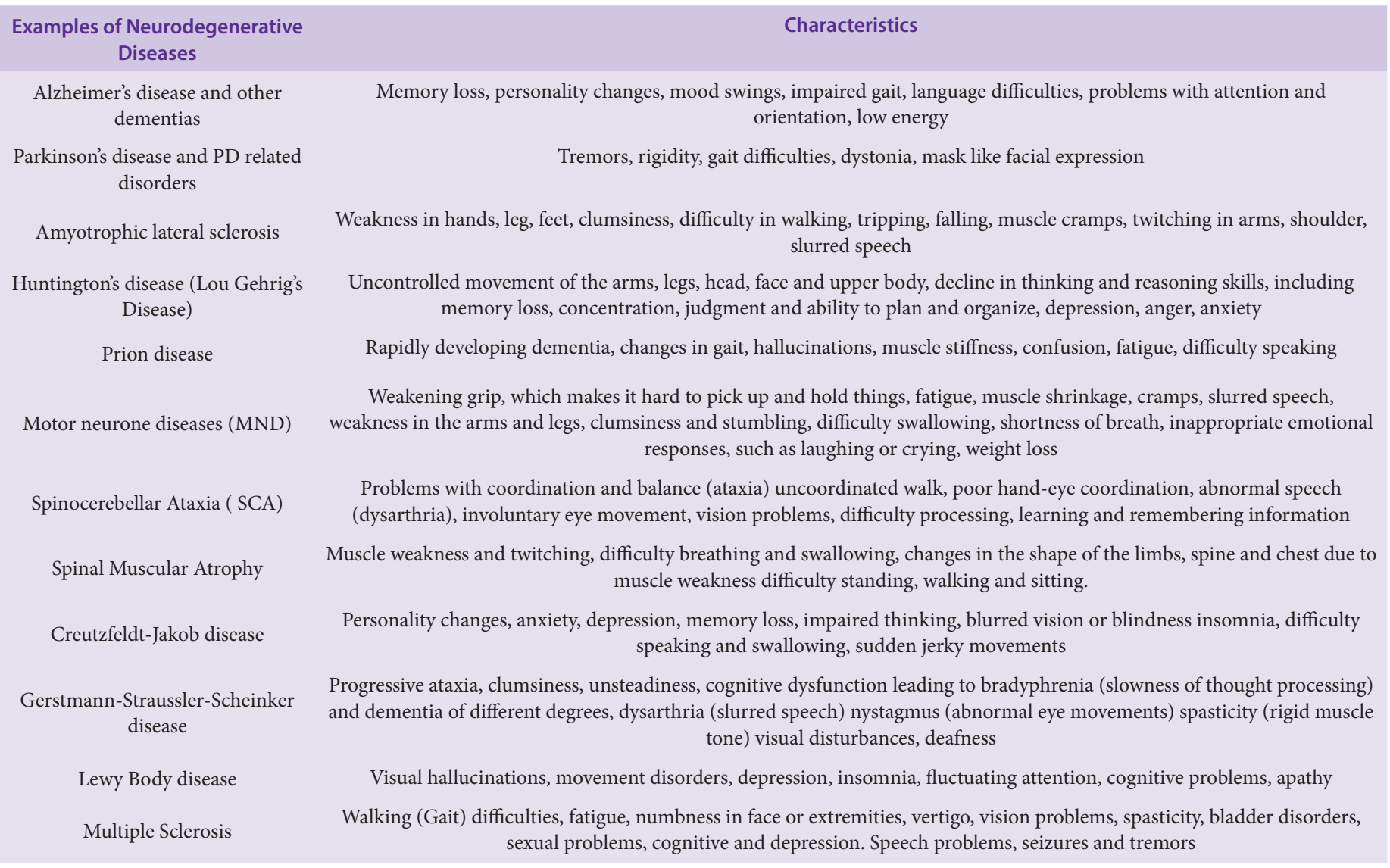

including cardiovascular disorders, muscle dysfunction, allergy and cancers. In addition to that, mitochondrial dysfunction and/or environmental factors strongly associated with age have also been a contributing factor in causing neurodegeneration..$^{5-57}$

Alzheimer's disease, the most common form of dementia, is one of the nation's largest public health crisis. Alzheimer's is known as an irreversible neurological condition that impairs cognition, orientation and functional capacity.$^{58}$ Moreover, it is one of the most common causes of fatality in the United States that cannot be prevented, cured or even slowed. The brain is an organ with high metabolism rate, oxidative stress is a common phenomenon in its neural tissue. ${ }^{59}$ The two main types of antioxidant compounds which are involved in oxidative stress regulation in the body are antioxidant enzymes, that neutralizes reactions against free radicals and reactive oxygen species and the other is antioxidant nutrients, which help as co-factors in catalytic activities. ${ }^{60,61}$ Some neurodegenerative disorders such as Alzheimer's, Parkinson's and Amyotrophic Lateral Sclerosis have a clear familial occurrence, suggesting a genetic basis. Among these affected families, the disease runs as an autosomal dominant trait, as in HD less frequently, the disease runs as an autosomal recessive trait, X-linked trait even a maternally inherited trait. Besides these "pure" genetic neurodegenerative diseases, others are sporadic but show a small contingent of patients in whom the illness is inherited. This is true for PD, AD and even ALS, of which about $10 \%$ of all cases are familial. In case of truly sporadic, which is the majority of patients; it appears that the genetic contribution to the neurodegenerative process is minimal. ${ }^{62}$ Most elderly individuals exhibit mild motor and cognitive alterations reminiscent of those found in neurodegeneration. This observation coincides with a popular believe that aging might be a "benign" form of neurodegeneration. This idea was supported and widely accepted until recently, that normal aging, like neurodegeneration, is inevitably associated with neuronal death. From as early as 1950s, decreased numbers of neurons in different regions of the brain were reported in aged humans with no overt neurological or psychiatric conditions which might have led to a neurodegenerative condition. Subsequent studies have estimated these losses to be as high as 100,000 neurons per day, which could easily explain the cognitive decline and decrease in brain weight traditionally associated with normal aging. ${ }^{63} \mathrm{~A}$ person might have a gene that makes him or her more susceptible to a certain neurodegenerative disease, but whether, when and how severely the person is affected depends on what environmental factors and lifestyle habits he or she is exposed to during life.

Inadequate nutrition and with existence of several diseases, cognitive function in 'healthy' individuals will be detrimentally fretful during their lifetime. Most people will encounter disease at some stage of their life, with majority of diseases associated with some form of negative consequences in cognitive function. ${ }^{69}$ However, over the years evidence is accumulating that cognitive decline during normal ageing can be attenuated by a wide variety of factors, such as improved nutrition, appropriate dietary supplementation, lifestyle modification with increased physical exercise and performance of mental exercises. ${ }^{70,71}$ Research work conducted with humans and on animals has demonstrated that the brain is sensitive to stress and is especially vulnerable during early childhood and old age. ${ }^{72,73}$ Exposure to Early-life Stress (ES) is associated with lasting changes in the structure of the adult brain and accelerated cognitive decline. Being exposed to stress of any kind in early life will in turn lead 
to unhealthy sedentary lifestyle which eventually affects the cognitive functions as it has been discussed above.

\section{Role of Free Radical in Development of Neurodegenerative Disorders}

Free radicals are metabolic by products produced naturally in our body through oxidation and it is said to be highly reactive and unstable molecules. In addition to being generated from a normal metabolic process in the body, it is also produced due to exposure to carcinogens and toxins in the environment such as ultraviolet light and tobacco smoke. ${ }^{74}$ The term oxidative stress is used to describe the oxidative damage resulting from unfavorable and critical imbalance between free radical generation and antioxidant defenses. ${ }^{75}$ Oxidative stress is a harmful condition that occurs when there is an excess of free radicals and/or a decrease in antioxidant levels. It can be the cause of environmental factors such as pollutants, chemicals and radiation. ${ }^{76}$ The brain, spinal cord and peripheral nerves which comprise the nervous system is rich in both iron and unsaturated fatty acids. The nervous tissue is particularly susceptible to oxidative damage due to high lipid content coupled with its high aerobic metabolic activity. There is substantial evidence that oxidative stress is a causative factor in the pathogenesis of major neurodegenerative diseases, including Parkinson's disease, Alzheimer's disease and amyotrophic lateral sclerosis (ALS, "Lou Gehrig's disease") also in cases of stroke, trauma and seizures. ${ }^{77}$ The proof to date for oxidative stress in Parkinson's Disease, Schizophrenia, Alzheimer's Disease and different neurodegenerative ailments is strongly persuasive. Clinical research exhibit that a range of events associated with Alzheimer's are capable of stimulating production of free radicals and depletion of antioxidant levels. Additionally, free radical injury is observed in the form of elevated lipid peroxidation and oxidation of DNA bases among patients with Parkinson's Disease. ${ }^{78}$

\section{CONCLUSION}

It is fundamental to understand the relationship between sedentary behaviour and health, modifying these behaviors in order to reduce the morbidity and mortality rates caused by the health complications which follow. Sedentary lifestyle leads to and exposes individuals (both old and young) to various non-communicable and devastating diseases. Neurodegenerative disorders remain an important source of morbidity and chronic suffering globally which has a strong link on lifestyle habits as discussed earlier. Hence, it is essential to promote healthy lifestyle, raising public awareness, understanding about diseases particularly on neurodegeneration and its possible root cause arising from one's lifestyle and habits particularly in the low socioeconomic community. Expansion of educational programs for general prediction, based on psychological empowerment in lower age groups (children and teenagers) could also be effective in reduction of drug abuses. Neurodegenerative disease can be associated with risk factors, for example, vascular risk factors (commonly hypertension and high cholesterol). Over the years we have come to know that most of the metabolic diseases specifically hypertension, diabetes and hypercholesterolemia are due to high consumption of high sodium substance commonly found in fast food. Frequent visits and excessive intake of processed or fast food are commonly practiced among the working urban community. According to most of the studies that has been done, it can be said that lifestyle has a significant influence on physical and mental health of humans. It is indeed crucial to encourage healthy behaviors, lifestyle modifications, physical and mental activities on a daily basis that contributes to cognitive ability and the maintenance of healthy mental functioning, such as exercising the mind, being social, healthy eating, stress reduction and appropriate sleep. It is also evident that in addition to reducing the risk of cognitive decline and dementia, these lifestyle modifications will delay the possible onset of neurodegeneration.

\section{CONFLICT OF INTEREST}

The authors declare no conflict of interest.

\section{ABBREVIATIONS}

AD: Alzheimer's disease; ES: Early-life stress; PD: Parkinson's disease; ROS: Reactive oxygen species; MCI: Mild cognitive impairment; VD: Vascular dementia; ALS: Amyotrophic lateral sclerosis; HD: Huntington's disease.

\section{REFERENCES}

1. Farhud DD. Impact of lifestyle on health. Iran J Public Health. 2015;44(11):1442 4.

2. Ziglio E, Currie C, Rasmussen VB. The WHO cross-national study of health behavior in school aged children from 35 countries: Findings from 2001-2002. J School Health. 2004;74(6):204-6.

3. Sundus M. The impact of using gadgets on children. J Depress Anxiety. 2018;7(1):1-3

4. Warburton DE, Nicol CW, Bredin SS. Health benefits of physical activity: The evidence. CMAJ. 2006;174(6):801-9.

5. Brannon L, Feist J, Updegraff JA. Health psychology: An introduction to behavior and health. $8^{\text {th }}$ ed., Cengage Learning. 2014.

6. Achalu El. Health Services and Health Care Delivery Systems. Port-Harcourt Pam Unique Publishing Co.Ltd. 2008.

7. Dunstan DW, Healy GN, Sugiyama T, Owen N. Too much sitting'and metabolic risk-Has modern technology caught up with us?. Eur Endocrinol. 2010;6(1):1923.

8. Salvatore D. World population trends and their impact on Economic Development. United State of America Green Wood Press. 2005;82.

9. WHO. "Physical Activity" Depression and anxiety exercise cases symptoms 2010.

10. Diaz KM, Shimbo D. Physical activity and the prevention of hypertension. Curr Hypertens Rep. 2013;15(6):659-68.

11. Cheung BM, Li C. Diabetes and hypertension: Is there a common metabolic pathway?. Curr Atheroscler Rep. 2012;14(2):160-6.

12. Fratiglioni L, Paillard-Borg S, Winblad B. An active and socially integrated lifestyle in late life might protect against dementia. Lancet Neurol. 2004;3(6):343 53

13. Rovio S, Spulber G, Nieminen LJ, Niskanen E, Winblad B, Tuomilehto J, et al. The effect of midlife physical activity on structural brain changes in the elderly. Neurobiol Aging. 2010;31(11):1927-36.

14. Komulainen $P$, Pedersen $M$, Hänninen $T$, Bruunsgaard $H$, Lakka TA, Kivipelto $M$, et al. BDNF is a novel marker of cognitive function in ageing women: The DR's EXTRA Study. Neurobiol Learn Mem. 2008;90(4):596-603.

15. Kern RZ, Brown AD. Disease adaptation may have decreased quality-of-life responsiveness in patients with chronic progressive neurological disorders. J Clin Epidemiol. 2004;57(10):1033-9.

16. Chekani F, Bali V, Aparasu RR. Quality of life of patients with Parkinson's disease and neurodegenerative dementia: A nationally representative study. Res Social Adm Pharm. 2016;12(4):604-13

17. Paulsen JS, Nance M, Kim JI, Carlozzi NE, Panegyres PK, Erwin C, et al. A review of quality of life after predictive testing for and earlier identification of neurodegenerative diseases. Prog Neurobiol. 2013;110:2-28.

18. Welsh MD. Measurement of quality of life in neurodegenerative disorders. Curr Neurol Neurosci Rep. 2001;1(4):346-9

19. Dunn AL, Andersen RE, Jakicic JM. Lifestyle physical activity interventions: History, short-and long-term effects and recommendations. Am J Prev Med. 1998;15(4):398-412

20. C3 collaborating for health. The benefits of physical activity for health and wellbeing. 2011. Available at: www.c3health.org/wwp-content/uploads/2009/09/ C3-review-of-physical-activity-and-health-v-1-20110603.pdf. last accessed on 01/03/2019

21. Dfarhud D, Malmir M, Khanahmadi M. Happiness and Health: The Biological Factors-Systematic Review Article. Iran J Public Health. 2014;43(11):1468-77.

22. Ancoli-Israel S, Ayalon L, Salzman C. Sleep in the elderly: normal variations and common sleep disorders. Harv Rev Psychiatry. 2008;16(5):279-86.

23. Bliwise D. Normal aging. Principles and Practice of Sleep Medicine. $4^{\text {th }}$ ed Philadelphia: Saunders. 2005:24-38

24. Caracciolo B, XuW, Collins S, Fratiglioni L. Cognitive decline, dietary factors and gut-brain interactions. Mech Ageing Dev. 2014;136:59-69.

25. Mozaffarian D, Hao T, Rimm EB, Willett WC, Hu FB. Changes in diet and lifestyle 
and long-term weight gain in women and men. N Eng Med J. 2011;364(25):2392404.

26. DeVos WM, DeVos EA. Role of the intestinal microbiome in health and disease: from correlation to causation. Nutr Rev. 2012;70(suppl_1):S45-56.

27. Bajaj JS, Ridlon JM, Hylemon PB, Thacker LR, Heuman DM, Smith S, et al. Linkage of gut microbiome with cognition in hepatic encephalopathy. Am J Physiol Gastrointest Liver Physiol. 2011;302(1):G168-75.

28. Murphy $T$, Thuret $S$. The systemic milieu as a mediator of dietary influence on stem cell function during ageing. Ageing Res Rev. 2015;19:53-64.

29. Ebadi M, Vahdanineya MS, Azin A, Aeeinparast A, Omidvari S, Jahangiri K. Prevalence of smoking: Health study of people's view of Iran. Payesh. 2011;10(3):365-72

30. Ekhtiari H, Behzadi A, Ganjahi H, Mokri A, Edalati H, Bakhtiari M, et al. Functional neuroimaging study of brain activation due to craving in heroin intravenous users. Iran J Psychiatry Clin Psychol. 2008;14(3):269-80.

31. Kuo CJ, Tsai SY, Liao YT, Conwell Y, Lin SK, Chang CL, et al. Risk and protective factors for suicide among patients with methamphetamine dependence: A nested case-control study. J Clin Psychiat. 2011;72(4):487-93

32. Clark DB, Martin CS, Cornelius JR. Adolescent-onset substance use disorders predict young adult mortality. J Adolesc Health. 2008;42(6):637-9.

33. Sh B, Mehrdad R, Akbari EH. A survey of Ecstasy use among $15-25$ year-olds in five areas of Tehran. TUMJ. 2008;65(11):49-54

34. Wheeler SB. Effects of self-esteem and academic performance on adolescent decision-making: An examination of early sexual intercourse and illegal substance use. J Adolesc Health. 2010;47(6):582-90

35. Thomée S, Härenstam A, Hagberg M. Mobile phone use and stress, sleep disturbances and symptoms of depression among young adults-a prospective cohort study. BMC Public Health. 2011;11(1):66-77.

36. Blank C, Leichtfried V, Schobersberger W, Möller C. Does leisure time negatively affect personal health?. World Leis J. 2015;57(2):152-7.

37. Salmon J, Tremblay MS, Marshall SJ, Hume C. Health risks, correlates and interventions to reduce sedentary behavior in young people. Am J Prev Med. 2011;41(2):197-206.

38. Iadecola C. The pathobiology of vascular dementia. Neuron. 2013;80(4):844-66.

39. Whatnall M, Collins C, Callister R, Hutchesson M. Associations between unhealthy diet and lifestyle behaviours and increased cardiovascular disease risk in young overweight and obese women. Healthcare. 2016;4(3):57.

40. WHO. Urbanization and Health. Bulletin of the World Health Organization 2010.

41. Biddle SJ, O'Connell S, Braithwaite RE. Sedentary behaviour interventions in young people: A meta-analysis. Br J Sports Med. 2011;45(11):937-42.

42. Block JP, He Y, Zaslavsky AM, Ding L, Ayanian JZ. Psychosocial stress and change in weight among US adults. Am J Epidemiol. 2009;170(2):181-92.

43. Biddle SJ, Pearson N, Ross GM, Braithwaite R. Tracking of sedentary behaviours of young people: A systematic review. Prev Med. 2010;51(5):345-51.

44. Gierach GL, Chang SC, Brinton LA, JrLacey JV, Hollenbeck AR, Schatzkin A, et al. Physical activity, sedentary behavior and endometrial cancer risk in the NIH-AARP Diet and Health Study. Int J Cancer. 2009;124(9):2139-47.

45. Alzheimer's A. Alzheimer's disease facts and figures. Alzheimer's and dementia. J Alzheimer's Assoc. 2015;11(3):332.

46. Salthouse TA. Does the level at which cognitive change occurs change with age?. Psychol Sci. 2012;23(1):18-23.

47. Salthouse T. Consequences of age-related cognitive declines. Annu Rev Psychol. 2012;63:201-26.

48. Yankner BA, LuT, Loerch P. The aging brain. Annu Rev Pathol. 2008;3:41-66.

49. Obeso JA, Stamelou M, Goetz CG, Poewe W, Lang AE, Weintraub D, et al. Past, present and future of Parkinson's disease: A special essay on the $200^{\text {th }}$ anniversary of the shaking palsy. Mov Disord. 2017;32(9):1264-310.

50. Salthouse TA. When does age-related cognitive decline begin?. Neurobiol Aging. 2009;30(4):507-14

51. Schaffer S, Asseburg H, Kuntz S, Muller WE, Eckert GP. Effects of polyphenols on brain ageing and Alzheimer's disease: Focus on mitochondria. Mol Neurobiol. 2012;46(1):161-78.

52. American Psychiatric Association. Diagnostic and statistical manual of mental disorders. BMC Med. 2013;17:133-7.
53. Cornejo VH, Hetz C. The unfolded protein response in Alzheimer's disease. Semin Immunopathol. 2013;35(3):277-92.

54. Zuo L, Koozechian MS, Chen LL. Characterization of reactive nitrogen species in allergic asthma. Ann Allergy Asthma Immunol. 2014;112(1):18-22.

55. Zuo L, Zhou T, Pannell BK, Ziegler AC, BestTM. Biological and physiological role of reactive oxygen species-the good, the bad and the ugly. Acta Physiologica. 2015;214(3):329-48.

56. He F, Zuo L. Redox roles of reactive oxygen species in cardiovascular diseases. Int J Mol Sci. 2015;16(11):27770-80.

57. Zuo L, Shiah A, Roberts WJ, Chien MT, Wagner PD, Hogan MC. Low PO conditions induce reactive oxygen species formation during contractions in single skeletal muscle fibers. Am J Physiol Regul Integr Comp Physiol. 2013;304(11):R1009-16.

58. Deerlin VM. The genetics and neuropathology of neurodegenerative disorders: perspectives and implications for research and clinical practice. Acta Neuropathological. 2012;124(3):297-303.

59. Bishop NA, Lu T, Yankner BA. Neural mechanisms of ageing and cognitive decline. Nature. 2010;464(7288):529-35.

60. Morris MC. Nutritional determinants of cognitive aging and dementia. Proceedings of the Nutrition Society. 2012;71(1):1-13.

61. Sardesai VM. Role of antioxidants in health maintenance. Nutr Clin Pract. 1995; 10(1):19-25.

62. Tanner CM, Ottman R, Goldman SM, Ellenberg J, Chan P, Mayeux R, et al. Parkinson disease in twins: An etiologic study. JAMA. 1999;281(4):341-6.

63. Finch CE, Day JR. Molecular biology of aging in the nervous system: A synopsis of the levels of mechanisms. Neurodegener Dis. 1994;33-50.

64. Alzheimer's Association. Alzheimer's disease facts and figures. Alzheimers Dement. 2016;12(4):459-509.

65. DeSilva HR, Khan NL, Wood NW. The genetics of Parkinson's disease. Curr Opin Genet Dev. 2000;10(3):292-8.

66. Albers DS, Beal MF. Mitochondrial dysfunction and oxidative stress in aging and neurodegenerative disease. J Neural Transm Suppl. 2000;59:133-54.

67. Matilla-Dueñas A, Ashizawa T, Brice A, Magri S, McFarland KN, Pandolfo M, et al. Consensus paper: Pathological mechanisms underlying neurodegeneration in spinocerebellar ataxias. Cerebellum. 2014;13(2):269-302.

68. Tolson D, RollandY, Andrieu S, Aquino JP, Beard J, Benetos A, et al. International Association of Gerontology and Geriatrics: A global agenda for clinical research and quality of care in nursing homes. J Am Med Dir Assoc. 2011;12(3):184-9.

69. Wesnes KA. Cognitive function testing: The case for standardization and automation. Br Menopause Soc J. 2006;12(4):158-63.

70. Ferreira N, Owen A, Mohan A, Corbett A, Ballard C. Associations between cog nitively stimulating leisure activities, cognitive function and age-related cognitive decline. Int J Geriatr Psychiatr. 2015;30(4):422-30.

71. Barberger-Gateau P. Nutrition and brain aging: How can we move ahead?. Eur J Clin Nutr. 2014;68(11):1245-49.

72. Costello EJ, Worthman C, Erkanli A, Angold A. Prediction from low birth weight to female adolescent depression: A test of competing hypotheses. Arch Gen Psychiatr. 2007;64(3):338-44.

73. Maselko J, Kubzansky L, Lipsitt L, Buka SL. Mother's affection at 8 months predicts emotional distress in adulthood. J Epidemiol Community Health 2011;65(7):621-5.

74. Lobo V, Patil A, Phatak A, Chandra N. Free radicals, antioxidants and functional foods: Impact on human health. Pharmacogn Rev. 2010;4(8):118-26.

75. Rock CL, Jacob RA, Bowen PE. Update on the biological characteristics of the antioxidant micronutrients: Vitamin C, vitamin E and the carotenoids. J Am Diet Assoc. 1996;96(7):693-702.

76. Liu Z, Zhou T, Ziegler AC, Dimitrion P, Zuo L. Oxidative stress in neurodegenerative diseases: From molecular mechanisms to clinical applications. Oxid Med Cell Longev. 2017:2017:2525967.

77. Ghadge GD, Lee JP, Bindokas VP, Jordan J, Ma L, Miller RJ, et al. Mutant superoxide dismutase-1-linked familial amyotrophic lateral sclerosis: Molecular mechanisms of neuronal death and protection. J Neurosci. 1997;17(22):8756-66.

78. Singh RP, Sharad S, Kapur S. Free radicals and oxidative stress in neurodegenerative diseases: relevance of dietary antioxidants. J Indian Acad Clin Med. $2004 ; 5(3): 218-25$

Cite this article: David AVA, Parasuraman S. Lifestyle Impact on Cognitive Decline and Neurodegeneration Overview. Free Radicals and Antioxidants. 2019;9(2):52-7. 